\title{
Enhanced Pavlovian conditioning with a change in appetitive reinforcer
}

\author{
JOHN M. PEARCE and EDWARD S. REDHEAD \\ Cardiff University, Cardiff, Wales
}

\begin{abstract}
In three experiments, rats received appetitive conditioning with two unconditioned stimuli (US+ and US*) that supported a similar conditioned response. Stimulus A was first paired with US+, and then, for a second stage of training, the compound $\mathrm{AB}$ was paired with US*. Subsequent test trials with A revealed an abnormally strong conditioned response. The findings from additional groups indicated that such enhanced conditioning with A depended on the presence of B in the second stage and on the use of US+ and US* for the two training stages. A modification to a configural theory of conditioning is proposed in order to explain the results.
\end{abstract}

In several experiments, the effect of pairing a conditioned and an unconditioned stimulus (CS and US) in the presence of a conditioned inhibitor that has signaled the omission of the US has been examined. Excitatory conditioning in these circumstances is generally more effective than when it takes place in the absence of the inhibitory stimulus. This effect, which has been referred to as either superconditioning (Rescorla, 1971) or supernormal conditioning (Wagner, 1971), has been demonstrated with both aversive (e.g., Rescorla, 1971; Wagner, 1971) and appetitive (Pearce \& Redhead, 1995) conditioning. Explanations for supernormal conditioning differ in detail (see, e.g., Pearce \& Redhead, 1995; Wagner \& Rescorla, 1972), but they share the common assumption that associative changes during compound conditioning will cease when the overall associative strength of the compound equals an asymptotic value that is set by the magnitude of the US. Thus, supernormal conditioning is effective because the excitatory CS must compensate for the negative influence of the inhibitory stimulus by acquiring abnormally high associative strength.

The purpose of the present experiments was to determine whether it is possible to obtain enhanced conditioning in conditions that are rather different from those that are normally used to demonstrate supernormal conditioning. The rationale for the experiments was based on the configural model of conditioning proposed by Pearce (1987, 1994). Consider an experiment in which the delivery of a US is first signaled by one stimulus, A, and then by a simultaneous compound of A with another stimulus, $\mathrm{B}$ - that is, $\mathrm{A}+/ \mathrm{AB}+$, where + refers to the US. During the first stage of training, the theory predicts that an association will develop between representations of A and the US. During the second stage, the addition of $\mathrm{B}$ is predicted to

This research was supported by a grant from the BBSRC of the United Kingdom. We thank Rob Honey for his many helpful comments concerning this work. Correspondence concerning this article should be addressed to J. M. Pearce, School of Psychology, Cardiff University, Cardiff, CF1 3YG, Wales (e-mail: pearcejm@cardiff.ac.uk). result in a generalization decrement, so that $\mathrm{AB}$ will elicit a weaker response than $\mathrm{A}$. Configural theory then predicts that a representation of $\mathrm{AB}$ in its entirety will enter into an association with the US, which will gain in strength until the associative strength of $\mathrm{AB}$, together with excitation that generalizes from $\mathrm{A}$ to $\mathrm{AB}$, is equal to an asymptotic value that is determined by the magnitude of the US. If A should now be presented by itself for a test trial, configural theory predicts that the original associative strength of $\mathrm{A}$, along with excitation that generalizes from $\mathrm{AB}$ to $\mathrm{A}$, will result in a stronger conditioned response $(C R)$ in the presence of $A$ than when conditioning takes place solely with $\mathrm{A}$. Thus, training of the sort $\mathrm{A}+/ \mathrm{AB}+$ is predicted to result in enhanced conditioning with $A$.

We have conducted a variety of unpublished experiments, in order to test the foregoing prediction, and the typical finding has been that responding during A was not augmented by the additional trials with $\mathrm{AB}$. In keeping with this conclusion, Rescorla (1999) has described several experiments that also failed to demonstrate any hint of enhanced conditioning with $\mathrm{A}$, using $\mathrm{A}+/ \mathrm{AB}+$ training. The purpose of the experiments reported below was to examine whether it is possible to obtain enhanced conditioning with A by using a slight modification to the $\mathrm{A}+/ \mathrm{AB}+$ design. In the first stage of training of each experiment, rats received conditioning in which $\mathrm{A}$ was paired with one appetitive US (say, a sucrose solution), and in the second stage, the compound $\mathrm{AB}$ was paired with a different appetitive US (say, food pellets) - that is, $\mathrm{A}+/ \mathrm{AB} *$. In stark contrast with the effects of training with $\mathrm{A}+$ / $\mathrm{AB}+$, this method of training revealed evidence of enhanced conditioning with $\mathrm{A}$. We postpone to the General Discussion section a consideration of why conditioning with $\mathrm{A}+/ \mathrm{AB}^{*}$, but not with $\mathrm{A}+/ \mathrm{AB}+$, should be effective in this way.

\section{EXPERIMENT 1}

For Stage 1 of the experiment, two groups received conditioning in which A was followed by the appetitive US 
of sucrose solution. For the second stage, Group $\mathrm{A}+/ \mathrm{AB} *$ received the simultaneous compound $\mathrm{AB}$, followed by a single food pellet, whereas for the other group, Group $\mathrm{A}+/ \mathrm{A}^{*}, \mathrm{~A}$ by itself signaled the food pellet. Test trials were then conducted in which A was presented in extinction to both groups. Providing that both reinforcers strengthen the same $\mathrm{CR}$, configural theory predicts that responding during $\mathrm{A}$ will be stronger by Group $\mathrm{A}+/ \mathrm{AB}$ * than by Group $A+/ A^{*}$. Additional nonreinforced trials with a third stimulus, C, were presented to both groups throughout the two conditioning stages.

\section{Method}

Subjects. The subjects were 32 naive male hooded Lister rats from the colony maintained at Cardiff University. They were approximately 3 months old at the start of the experiment. Prior to the experiment, they were gradually reduced to $80 \%$ of their free-feeding weights by food deprivation and were maintained at this level throughout the experiment by being fed a restricted amount of food after each experimental session. The rats were housed in a lightproof room in which the lights were on for $14.5 \mathrm{~h}$ each day. They were trained on successive days, at the same time, during the period when the lights were on in their holding room. The rats were randomly assigned to the two groups at the start of the experiment.

Apparatus. Eight identical conditioning chambers $(24.5 \times 23.0$ $\times 20.0 \mathrm{~cm}$ ) were housed in separate environmental isolation chests. An exhaust fan in each of the chests provided masking noise of $71 \mathrm{~dB}$. Three walls of each chamber were constructed from aluminum, the front wall was clear Perspex, and the ceiling was opaque Perspex. The floor was constructed from steel rods, $6.4 \mathrm{~mm}$ in diameter, which were spaced $1.9 \mathrm{~cm}$ center to center and ran parallel to the wall containing the magazine. A 5- $\omega$ speaker located on the front wall delivered white noise at an intensity of $76 \mathrm{~dB}$, a 5- $\omega$ speaker located in the middle of the upper half of the rear wall delivered a $2-\mathrm{kHz}$ tone at an intensity of $76 \mathrm{~dB}$, and a $5-\omega$ speaker in the middle of the lower half of the rear wall delivered a $7-\mathrm{Hz}$ click at an intensity of $80 \mathrm{~dB}$. There was a $5 \times 6 \mathrm{~cm}$ recessed food magazine, into which 45-mg food pellets (traditional formula, P. J. Noyes, Lancaster, $\mathrm{NH}$ ) could be delivered, which was located in the front wall, with its base $0.5 \mathrm{~cm}$ above the grid floor. To gain access to the magazine, the subjects were required to push open a clear Perspex flap, which was hinged at the top. A peristaltic pump was used to dispense $0.5 \mathrm{ml}$ of $20 \%$ sucrose solution into an indentation in the base of the magazine. A $10-\mathrm{mm}$-deep rectangular frame was attached to the front wall of the chamber around the opening to the recessed food magazine. Three pairs of photodiode sensors were set into this frame in such a position that horizontal infrared beams $5 \mathrm{~mm}$ in front of the closed magazine flap were located 10,20, and $30 \mathrm{~mm}$ above the grid floor. Appropriate circuitry allowed the interruption of these beams to be detected by the computer that controlled experimental events.

Procedure. All the rats were given two 30-min sessions of magazine training in which a single pellet of food was delivered according to a variable time (VT) 60 -sec schedule in the first session and $0.5 \mathrm{ml}$ of sucrose solution was delivered according to the same schedule in the second session. The magazine flap was taped open for the first session, whereas for the rest of the experiment, it was free.

During Stage 1 of the experiment, both groups received nine sessions of conditioning in which CS A, the delivery of white noise for $10 \mathrm{sec}$, was followed immediately by the delivery of $0.5 \mathrm{ml}$ of sucrose solution. There were also trials with the clicker presented for $10 \mathrm{sec}$, followed by nothing. Within each session, there were 12 trials with each stimulus presented in a random sequence, with the constraint that no more than 3 trials of the same type could occur in succession. The duration of a session was $52 \mathrm{~min}$, and the interval from the end of one presentation of a stimulus to the start of the next presentation was determined by a VT 120 -sec schedule (range, $60-180 \mathrm{sec}$ ).

In Stage 2, Group A+/A* received the same treatment as that administered for the first stage, except that the US presented after $\mathrm{CS}$ A was a single pellet of food. Group A+/AB* received the same training as Group $\mathrm{A}+/ \mathrm{A}^{*}$, but each presentation of $\mathrm{A}$ was accompanied by the tone, CS B, for $10 \mathrm{sec}$. There were four complete sessions in Stage 2, and the timing of the trials was the same as that for Stage 1 . The test session, which took place on the day following the completion of Stage 2, consisted of 12 extinction trials in which CS A was presented by itself and was not followed by a reinforcer. A VT 120-sec schedule was used to determine the timing of successive presentations of CS A.

The measure of conditioning in every experiment was magazine activity, which was deemed to be taking place whenever at least one of the infrared beams in front of a food magazine was broken. Throughout the experiment, magazine activity was recorded for $10 \mathrm{sec}$ prior to each trial and during each trial. The total time during which the beams were broken in the initial $10 \mathrm{sec}$ was subtracted from the total time they were broken during the trial, to provide a difference score as the measure of magazine activity. A significance level of .05 was adopted for all of the experiments.

\section{Results and Discussion}

Conditioning progressed as expected during the first stage of training. In the final session of this stage, the mean difference scores in the presence of $\mathrm{A}$ and $\mathrm{C}$, respectively, were 7.0 and $1.3 \mathrm{sec}$ for Group $\mathrm{A}+/ \mathrm{A}^{*}$ and 7.2 and $1.3 \mathrm{sec}$ for Group $\mathrm{A}+/ \mathrm{AB}^{*}$.

The left-hand panel of Figure 1 shows the group mean difference scores for each of the four sessions of training in Stage 2 in the presence of $\mathrm{AB}$ and $\mathrm{C}$ for Group $\mathrm{A}+/$ $\mathrm{AB}^{*}$ and in the presence of $\mathrm{A}$ and $\mathrm{C}$ for Group $\mathrm{A}+/ \mathrm{A}^{*}$. An analysis of variance (ANOVA) of individual mean difference scores that were recorded during the reinforced trials of the final session of Stage 2 indicated that there was not a significant difference between the groups $(F<1)$.

The right-hand panel of Figure 1 shows the mean difference scores that were recorded in the presence of CS A, in 2-trial blocks, for both groups during the 12 extinction test trials. Throughout the test stage, the duration of magazine activity in the presence of $\mathrm{A}$ was greater for Group $\mathrm{A}+/ \mathrm{AB}^{*}$ than for Group $\mathrm{A}+/ \mathrm{A}^{*}$. The results from a twoway ANOVA of individual mean difference scores for successive 2-trial blocks revealed a significant effect of group $[F(1,30)=4.78]$ and of trial block $[F(5,150)=$ 13.30], but the interaction was not significant $(F<1)$.

The mean duration of magazine activity during the pre-CS periods of the 12 extinction test trials was $0.3 \mathrm{sec}$ for Group $\mathrm{A}+/ \mathrm{A}^{*}$ and $0.4 \mathrm{sec}$ for Group $\mathrm{A}+/ \mathrm{AB}^{*}$. An ANOVA, similar to that conducted with the difference scores for the test session, revealed that the effect of group and the group $\times$ trial block interaction were not significant $\left(F_{\mathrm{S}}<1\right)$ but that there was a significant effect of trial block $[F(5,150)=4.7]$.

Throughout the extinction trials with A, Group A+/ $\mathrm{AB}^{*}$ engaged in more magazine activity than did Group $\mathrm{A}+/ \mathrm{A}^{*}$. Thus, by conducting conditioning with $\mathrm{A}$ in Stage 1 and with the compound AB in Stage 2, it was possible to augment responding to $\mathrm{A}$ on the test trials to a 
STAGE 2

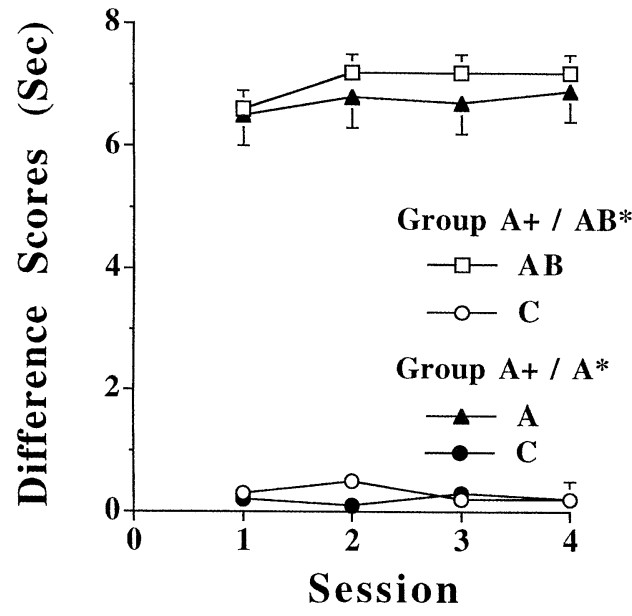

TEST

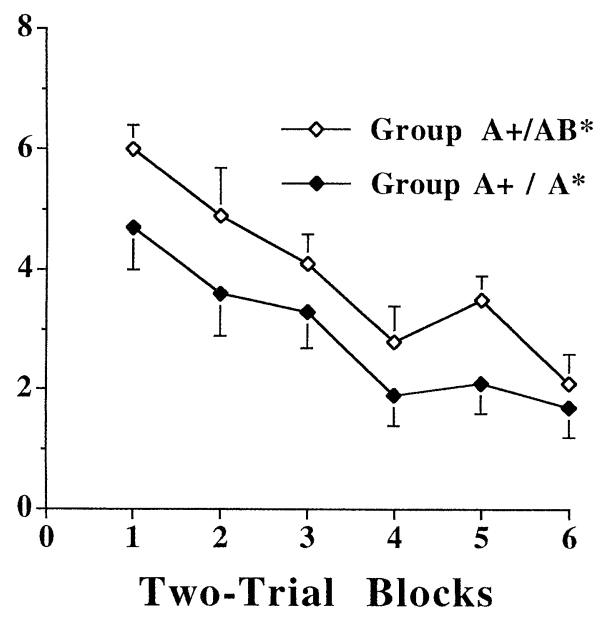

Figure 1. The group mean difference scores, with standard error bars, for the reinforced and nonreinforced conditioning trials of the second stage of conditioning (left-hand panel) and for the extinction test trials with A (right-hand panel) for the two groups of Experiment 1.

greater extent than when conditioning was conducted solely with $\mathrm{A}$ in both stages of the experiment.

\section{EXPERIMENT 2}

According to the configural theory of Pearce (1987, 1994), enhanced conditioning with A in the previous experiment was a consequence of the generalization decrement that was engendered by the addition of $\mathrm{B}$ for the $\mathrm{AB}^{*}$ trials in Stage 2. As was noted in the introduction, the theory predicts that it should also be possible to observe this effect with A when the same reinforcer is used during the first two conditioning stages. In contrast with this prediction, results from our unpublished studies, as well as findings by Rescorla (1999), suggest that enhanced conditioning with $\mathrm{A}$ is unlikely to result from the $\mathrm{A}+/ \mathrm{AB}+$ treatment. One purpose of the present experiment, therefore, was to determine whether enhanced responding in the presence of $\mathrm{A}$, after conditioning with $\mathrm{A}$ and then $\mathrm{AB}$, depends on the use of a different US for the two stages of conditioning. Another reason for conducting the experiment was to assess whether the effect demonstrated in Experiment 1 could be obtained with a withingroup, rather than a between-group, methodology.

There were two groups in Experiment 2. Group $\mathrm{A}+/$ $\mathrm{AB}^{*}$ first received trials in which stimuli $\mathrm{A}$ and $\mathrm{C}$ were each paired with the same US. For Stage 2 , the compound $A B$ was then paired with a different US, whereas $C$ was again paired with the same US as that for Stage 1 . The two stimuli $\mathrm{A}$ and $\mathrm{C}$ were then presented in extinction for a series of test trials. If the effect shown in the first experiment could be found with a within-group design, the training should result in enhanced conditioning with $\mathrm{A}$, and responding during the test trials with this stimulus will be stronger than that with $\mathrm{C}$. In order to determine whether the change of US at the outset of Stage 2 is necessary for this outcome, Group $\mathrm{A}+/ \mathrm{AB}+$ was treated in much the same way as Group $\mathrm{A}+/ \mathrm{AB}^{*}$, except that $\mathrm{A}$ and $\mathrm{AB}$ were paired with the same US. If the results from previous experiments (e.g., Rescorla, 1999) are reliable, the strength of responding during A will not be stronger than during $\mathrm{C}$ throughout the test phase for Group $\mathrm{A}+$ / $\mathrm{AB}+$. In addition, responding during $\mathrm{A}$ should be stronger for Group $A+/ A B *$ than for Group $A+/ A B+$. Finally, in order to equate the experience of the two groups, as far as exposure to the two reinforcers was concerned, for Group $\mathrm{A}+/ \mathrm{AB}+$, the control stimulus, $\mathrm{C}$, was paired with US + in Stage 1 and with US* in Stage 2.

\section{Method}

Subjects and Apparatus. The subjects were 24 male hooded Lister rats from the same stock and maintained in the same manner as those for Experiment 1. The apparatus was the same as that for Experiment 1. Prior to the start of the experiment, the rats were randomly assigned in equal numbers to the two groups.

Procedure. Following magazine training, there were eight sessions of conditioning for Stage 1. In each session, twelve 10-sec trials with CS A were intermixed randomly with twelve 10 -sec trials with CS C. For half the rats in each group, a single food pellet was delivered after $\mathrm{A}$ and $\mathrm{C}$, whereas for the remaining rats, sucrose solution was delivered after these stimuli. Half the rats in each of these subgroups received the tone as CS A and the noise as CS C, whereas the remainder received the noise as CS A and the tone as CS C.

The four sessions of training in Stage 2 was based on that used for Stage 1, with the following changes. For both groups, every presentation of A was accompanied simultaneously by CS B, the 7-Hz click. In addition, the US that followed $\mathrm{AB}$ in Group $\mathrm{A}+/ \mathrm{AB}^{*}$ and $\mathrm{C}$ in Group $\mathrm{A}+/ \mathrm{AB}+$ was opposite to the one used for Stage 1 . The test session comprised six trials, with each of $\mathrm{A}$ and $\mathrm{C}$ presented in an alternating sequence in extinction. For half the rats in each group, the first trial of the sequence was with A. Procedural details from any phase of the experiment that have been omitted were the same as those for Experiment 1. 

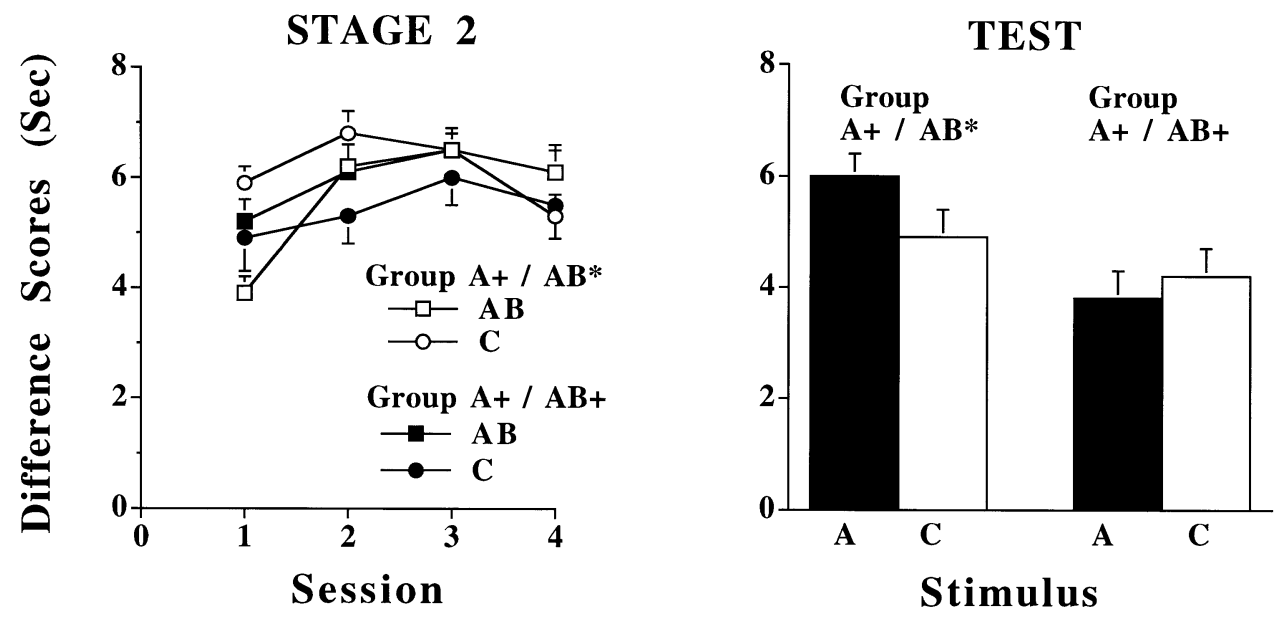

Figure 2. The group mean difference scores, with standard error bars, for the conditioning trials during the second stage of conditioning (left-hand panel) and for the extinction test trials with $A$ and with $C$ (right-hand panel) for the two groups of Experiment 2.

\section{Results and Discussion}

Conditioning in the first two stages progressed as expected. In the final session of Stage 1, the mean difference scores in the presence of $\mathrm{A}$ and $\mathrm{C}$, respectively, were 5.9 and $6.4 \mathrm{sec}$ for Group $\mathrm{A}+/ \mathrm{AB}^{*}$ and 6.2 and $5.8 \mathrm{sec}$ for Group $\mathrm{A}+/ \mathrm{AB}+$. The left-hand panel of Figure 2 shows the group mean difference scores for the four sessions of Stage 2. By the end of this stage, both groups were performing similarly in the presence of A and C. An ANOVA of individual mean difference scores for the final session of Stage 2 revealed that the effects of stimulus [A or C; $F(1,22)=1.28]$ and group $[F(1,22)=1.47]$ and the interaction $(F<1)$ were not significant.

Figure 2 shows, for both groups, the mean difference scores for the six test trials combined with $\mathrm{A}$ and with $\mathrm{C}$. Group $\mathrm{A}+/ \mathrm{AB}^{*}$ spent more time engaged in magazine activity in the presence of $A$ than of $C$. In contrast, there is a slight suggestion that responding was weaker during $\mathrm{A}$ than during $\mathrm{C}$ in Group $\mathrm{A}+/ \mathrm{AB}+$. The figure also shows that Group $\mathrm{A}+/ \mathrm{AB}^{*}$ engaged in more magazine activity in the presence of $\mathrm{A}$ than did Group $\mathrm{A}+/ \mathrm{AB}+$. A two-way ANOVA, with the factors of group and stimulus, revealed a significant group $\times$ stimulus interaction $[F(1,22)=$ $4.33]$ and a significant effect of group $[F(1,22)=7.3]$, but the effect of stimulus was not significant $(F<1)$. Subsequent simple main effects analyses revealed that the mean difference scores for the six test trials with $\mathrm{A}$ were significantly greater than those with $\mathrm{C}$ in Group $\mathrm{A}+/ \mathrm{AB}^{*}$ $[F(1,22)=4.64]$, but not in Group $\mathrm{A}+/ \mathrm{AB}+(F<1)$. Furthermore, the mean difference scores for the six test trials for Group $\mathrm{A}+/ \mathrm{AB} *$ were significantly greater than those for Group $\mathrm{A}+/ \mathrm{AB}+$ in the presence of $\mathrm{A}[F(1,44)=11.52]$, but not of $\mathrm{C}[F(1,44)=1.37]$.

The mean duration of activity during the combined 12 pre-CS periods of the test session was $0.4 \mathrm{sec}$ for Group
$\mathrm{A}+/ \mathrm{AB}^{*}$ and $0.7 \mathrm{sec}$ for Group $\mathrm{A}+/ \mathrm{AB}+$. This difference between the groups was not significant $[F(1,22)=2.7]$.

The results from Group $\mathrm{A}+/ \mathrm{AB}^{*}$ replicate the findings from the first experiment by showing that pairing $\mathrm{A}$ with US+ and then $\mathrm{AB}$ with US* results in enhanced conditioning with $\mathrm{A}$. In the case of the present experiment, this effect was revealed with a within-group methodology in which a control stimulus had been paired with the same US for both stages of the experiment. The results from the second group emphasize the importance of the change of US for enhanced conditioning with A. Group $\mathrm{A}+/ \mathrm{AB}+$ received similar training to that for Group $\mathrm{A}+/$ $\mathrm{AB}^{*}$, except that the same US was used for both stages of training and, on this occasion, there was no evidence of an exceptionally strong $\mathrm{CR}$ during the test trials with $\mathrm{A}$. The results from Group $\mathrm{A}+/ \mathrm{AB}+$ do not support predictions that can be derived from the configural theory of Pearce $(1987,1994)$, but they are consistent with results from our unpublished experiments, as well as with findings reported by Rescorla (1999).

\section{EXPERIMENT 3}

Although the results from Group $\mathrm{A}+/ \mathrm{AB}+$ are not in keeping with predictions that can be derived from a configural theory of conditioning (Pearce, 1987, 1994), the results from Group $\mathrm{A}+/ \mathrm{AB}^{*}$ are consistent with this theory. Experiment 3 was conducted in order to test a prediction from configural theory concerning the circumstances that determine whether or not $\mathrm{A}+/ \mathrm{AB}^{*}$ training will result in enhanced conditioning with $\mathrm{A}$. The procedure for the four groups of the experiments is summarized in Table 1. Group $\mathrm{A}+/ \mathrm{AB}^{*}$ received training similar to that of its namesakes in the previous studies. Thus, during Stage 1, A was paired with US+, whereas in Stage 2, AB 
Table 1 Summary of the Design for Experiment 3

\begin{tabular}{lllc}
\hline \multicolumn{1}{c}{ Group } & Stage 1 & Stage 2 & Test \\
\hline $\mathrm{A}+/ \mathrm{AB}^{*}$ & $\mathrm{~A}+, \mathrm{C}^{*}$ & $\mathrm{AB}^{*}, \mathrm{C}^{*}$ & $\mathrm{~A}$ \\
$\mathrm{~A}+\mathrm{B}^{*} / \mathrm{AB}^{*}$ & $\mathrm{~A}+, \mathrm{B}^{*}$ & $\mathrm{AB}^{*}, \mathrm{~B}^{*}$ & $\mathrm{~A}$ \\
$\mathrm{~A}+/ \mathrm{AB}+$ & $\mathrm{A}+, \mathrm{C}^{*}$ & $\mathrm{AB}+\mathrm{C}+$ & $\mathrm{A}$ \\
$\mathrm{A}+\mathrm{B}^{*} / \mathrm{AB}+$ & $\mathrm{A}+, \mathrm{B}^{*}$ & $\mathrm{AB}+, \mathrm{B}+$ & $\mathrm{A}$ \\
\hline
\end{tabular}

Note-For an explanation of the symbols, see the text.

was paired with US*. Test trials with A were expected to reveal evidence of enhanced conditioning. Group $\mathrm{A}+\mathrm{B} * /$ $\mathrm{AB}^{*}$ was treated in a similar way to Group $\mathrm{A}+/ \mathrm{AB}^{*}$, except that additional $\mathrm{B}^{*}$ conditioning trials were included in both stages of the experiment. In order to ensure that both groups received similar exposure to the two reinforcers, Group $\mathrm{A}+/ \mathrm{AB}^{*}$ received $\mathrm{C}^{*}$ trials during the first two stages of the experiment.

At the outset of Stage 2, when the compound $A B$ is first presented to Group $\mathrm{A}+\mathrm{B}^{*} / \mathrm{AB}^{*}$, configural theory predicts that the training given with $\mathrm{A}$ and $\mathrm{B}$ individually will enable each of these stimuli to provide a source of excitation that will generalize to AB. Moreover, because A and $B$ each provide half of the elements of $A B$, configural theory predicts that half of their associative strengths will generalize to the compound. The combination of these two sources of excitation will then lead to the compound's arousing asymptotic excitation, and there will be no need for the configural representation activated by $\mathrm{AB}$ to enter into an excitatory association. When A is subsequently presented for testing, there will be no generalization from $\mathrm{AB}$ to bolster the excitatory properties originally acquired by $\mathrm{A}$, and there should be no evidence of enhanced conditioning with this stimulus. Thus, configural theory predicts that $\mathrm{A}+/ \mathrm{AB}^{*}$ training will not result in enhanced conditioning with $\mathrm{A}$, if additional $\mathrm{B} *$ trials are included in the two stages of training.

The remaining two groups in the experiment received the same training as that for the two groups just described during Stage 1, but during Stage 2, the US presented after $\mathrm{AB}, \mathrm{B}$, and $\mathrm{C}$, was the same as that presented after $\mathrm{A}$ in Stage 1. Thus, Group $\mathrm{A}+/ \mathrm{AB}+$ received conditioning with $\mathrm{A}+$ and $\mathrm{C}^{*}$ in Stage 1 and with $\mathrm{AB}+$ and $\mathrm{C}+$ in Stage 2. Although configural theory predicts that test trials with A will reveal evidence of enhanced conditioning, the results from the previous experiment indicate that this prediction will not be confirmed. Thus, the inclusion of Group $\mathrm{A}+/ \mathrm{AB}+$ allows the reliability of the results from the group of the same name in Experiment 2 to be assessed with a slightly different method of training and testing. Finally, Group $\mathrm{A}+\mathrm{B} * / \mathrm{AB}+$ received conditioning with $\mathrm{A}+$ and $\mathrm{B} *$ in Stage 1 and with $\mathrm{AB}+$ and $\mathrm{B}+$ in Stage 2. Quite apart from the effect of pairing $A$ and $A B$ with the same US, configural theory predicts that enhanced conditioning will not be seen in Group $A+B *$ / $\mathrm{AB}+$. The reasons for this prediction are the same as those for Group $\mathrm{A}+\mathrm{B} * / \mathrm{B}^{*}$.

\section{Method}

Subjects and Apparatus. The 64 rats were from the same stock and were maintained in the same manner as those in Experiment 1. The conditioning chambers were the same as those used for Experiment 1 . The experiment was conducted in two replications. At the start of each replication, 8 rats were assigned at random to each of the four groups.

Procedure. Upon the completion of two sessions of magazine training, which was conducted in a similar fashion to that for Experiment 1 , the four groups were given nine sessions of training in Stage 1 of the experiment. In each session, for every group, there were 12 conditioning trials with CS A paired with one US and 12 trials with a second CS paired with the other US. The second CS was $C S B$, the clicker, for Groups $A+B * / A B *$ and $A+B * / A B+$ and $\mathrm{CS} \mathrm{C}$, the tone, for Groups $\mathrm{A}+/ \mathrm{AB}^{*}$ and $\mathrm{A}+/ \mathrm{AB}+$. For half the rats in each group, CS A was paired with a food pellet, and for the other half, it was paired with sucrose solution. For all the rats, A was white noise, B was a clicker, and C was a tone. Details of these stimuli can be found in Experiment 1 .

In each of the four sessions of Stage 2, all the groups received 12 conditioning trials with compound $\mathrm{AB}$ and 12 trials with a single $\mathrm{CS}$. The same US was presented on both types of trial. For Groups $\mathrm{A}+\mathrm{B}^{*} / \mathrm{AB}^{*}$ and $\mathrm{A}+/ \mathrm{AB}^{*}$, the US was opposite to the one that was paired with CS A in the first stage of the experiment, whereas for the remaining two groups, the US presented in Stage 2 was the same as the one paired with A in Stage 1. The single CS that was presented in Stage 2 was $C$ for Groups $A+/ A B^{*}$ and $A+/ A B+$ and $B$ for Groups $\mathrm{A}+\mathrm{B}^{*} / \mathrm{AB}^{*}$ and $\mathrm{A}+\mathrm{B}^{*} / \mathrm{AB}+$. All the groups then received 8 nonreinforced test trials with CS A presented alone in a final test session. Procedural details that have been omitted were the same as those for Experiment 1

\section{Results}

By the final session of Stage 1, all four groups responded similarly during both types of trial. The mean difference scores in this session that were recorded in the presence of $\mathrm{A}$ and $\mathrm{C}$, respectively, were 4.8 and $4.9 \mathrm{sec}$ for Group $\mathrm{A}+/ \mathrm{AB}^{*}$ and 4.1 and $4.3 \mathrm{sec}$ for Group $\mathrm{A}+/ \mathrm{AB}+$; for the remaining two groups, the mean difference scores in the presence of $A$ and $B$, respectively, were 4.6 and $4.0 \mathrm{sec}$ for Group $\mathrm{A}+\mathrm{B}^{*} / \mathrm{AB}^{*}$ and 4.7 and $4.8 \mathrm{sec}$ for Group $\mathrm{A}+\mathrm{B} * / \mathrm{AB}+$.

The left-hand panel of Figure 3 shows the group mean difference scores for the four sessions of conditioning with AB in Stage 2. There is no clear evidence of stronger responding when $\mathrm{AB}$ was composed of two stimuli that had both previously been paired with a US (Groups $\mathrm{A}+\mathrm{B} * / \mathrm{AB}^{*}$ and $\left.\mathrm{A}+\mathrm{B} * / \mathrm{AB}+\right)$ than when it was composed of only one element that had previously been paired with a US (Groups $\mathrm{A}+/ \mathrm{AB}^{*}$ and $\mathrm{A}+/ \mathrm{AB}+$ ). A two-way ANOVA of individual difference scores for the final session of Stage 2, with the factors of reinforcer (whether or not $\mathrm{AB}$ was followed by the same US that had previously been paired with $A$ ) and pretraining (whether or not $B$ had been presented for conditioning in Stage 1), revealed that both main effects and the interaction were not significant $(F \mathrm{~s}<1)$.

The group mean difference scores for the eight extinction test trials combined are shown in the right-hand panel of Figure 3. Overall, the duration of the scores was greater for Group $\mathrm{A}+/ \mathrm{AB}^{*}$ than for any other group. $\mathrm{A}$ 

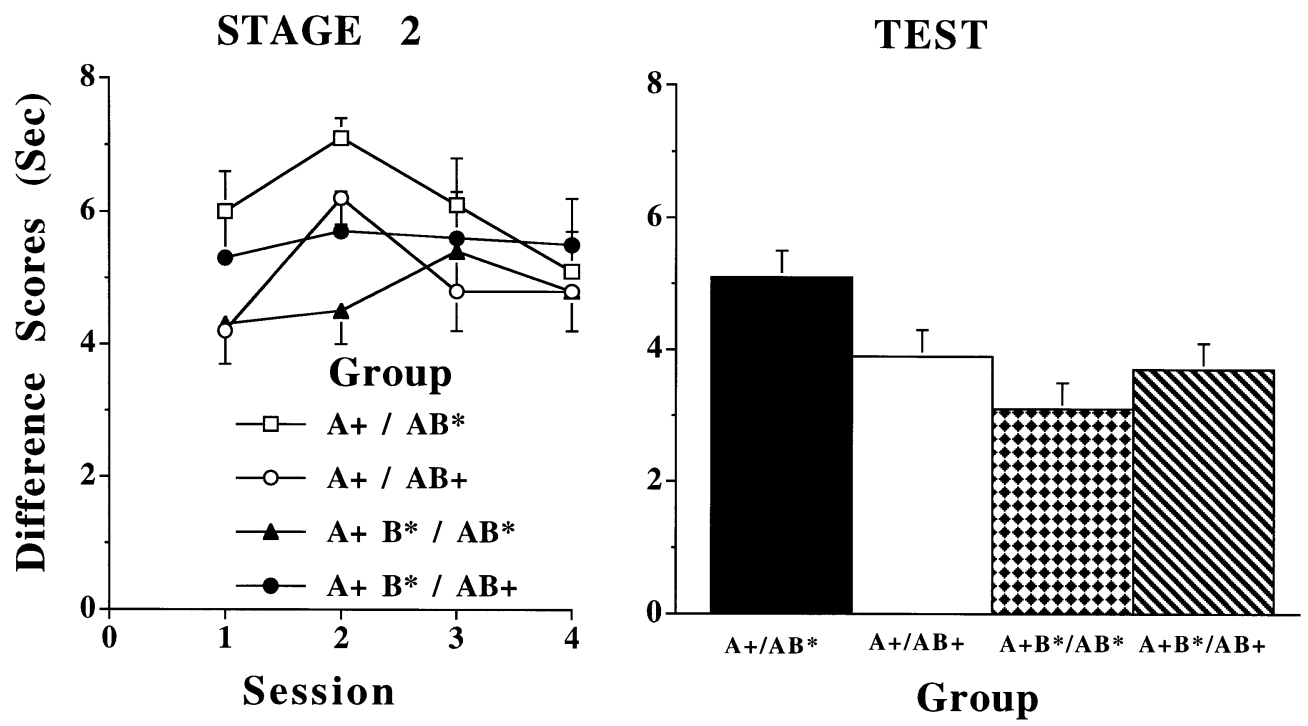

Figure 3. The group mean difference scores, with standard error bars, for the conditioning trials with AB during the second stage of conditioning (left-hand panel) and for the extinction test trials with A (righthand panel) for the four groups of Experiment 3.

two-way ANOVA, using the factors described in the previous paragraph of reinforcer and pretraining with $\mathrm{B}$, revealed that the effect of reinforcer was not significant $(F<1)$, but that the effect of pretraining $[F(1,56)=7.2]$ and the interaction $[F(1,56)=5.57]$ were significant. Subsequent simple effects analyses then revealed that the difference scores were significantly longer for Group $\mathrm{A}+/ \mathrm{AB} *$ than for either Group $\mathrm{A}+/ \mathrm{AB}+[F(1,56)=4.26]$ or Group $\mathrm{A}+\mathrm{B}^{*} / \mathrm{AB}^{*}[F(1,56)=12.7]$. The differences between Group $\mathrm{A}+\mathrm{B} * / \mathrm{AB}+$ and Group $\mathrm{A}+\mathrm{B} * / \mathrm{AB} *$ $[F(1,56)=1.62]$ and between Group $\mathrm{A}+/ \mathrm{AB}+$ and Group $\mathrm{A}+\mathrm{B} * / \mathrm{AB}+(F<1)$ were not significant.

The mean duration of magazine activity during the pre-CS periods of the test session, for all the trials combined, were $0.8 \mathrm{sec}$ for Group $\mathrm{A}+\mathrm{B}^{*} / \mathrm{AB}^{*}, 1.0 \mathrm{sec}$ for Group $\mathrm{A}+\mathrm{B} * / \mathrm{AB}+$, and $1.1 \mathrm{sec}$ for the remaining two groups. A two-way ANOVA of individual mean durations of magazine activity, similar to the one just described, revealed that both main effects and the interaction were not significant $\left(F_{\mathrm{S}}<1\right)$.

An unexpected feature of the results shown in Figure 3 is that, at the outset of Stage 2, there was a considerable difference among the mean durations of magazine activity for the four groups. These differences among the groups either were not evident or were considerably smaller in the final session of the previous stage. In the final session of Stage 1, the mean durations of magazine activity in the presence of $\mathrm{A}$ and the other stimulus that was presented for conditioning in this stage (B or $\mathrm{C}$ ), respectively, were 4.8 and $4.9 \mathrm{sec}$ for Group $\mathrm{A}+/ \mathrm{AB}^{*}, 4.6$ and $4.0 \mathrm{sec}$ for Group $\mathrm{A}+\mathrm{B}^{*} / \mathrm{AB}^{*}, 4.1$ and $4.3 \mathrm{sec}$ for Group $\mathrm{A}+/ \mathrm{AB}+$, and 4.7 and $4.8 \mathrm{sec}$ for Group $\mathrm{A}+\mathrm{B}^{*} /$ $\mathrm{AB}+$. A three-way ANOVA of individual mean durations of magazine activities for the final session of Stage 1 re- vealed that the main effects of reinforcer (whether or not AB in Stage 2 was paired with the same US that was paired with A in Stage 1), pretraining (whether or not B was presented for conditioning in Stage 1), and stimulus were not significant $\left(F_{\mathrm{S}}<1\right)$. In addition, the interactions among these factors were not significant (all $F \mathrm{~s}<1$ ), except for the reinforcer $\times$ pretraining interaction $[F(1,60)=1.3, p>.25]$. In view of these results, we are unable to offer with confidence any convincing explanation for the pattern of responding that was observed at the outset of Stage 2. Moreover, since the performance of the four groups was very similar at the end of this stage, it is difficult to attribute the results from the test trials to responding at the outset of Stage 2 .

\section{Discussion}

The results from Groups $\mathrm{A}+/ \mathrm{AB} *$ and $\mathrm{A}+/ \mathrm{AB}+$ are consistent with the findings from Experiment 2, in showing more persistent magazine activity during the test trials with $\mathrm{A}$ in the former than in the latter group. Thus, once again, our results demonstrate that enhanced conditioning with $\mathrm{A}$, after conditioning with $\mathrm{A}$ and with $\mathrm{AB}$, depends on the use of a different US for the two stages of conditioning. A novel finding from the experiment concerns Group $\mathrm{A}+\mathrm{B}^{*} / \mathrm{AB}^{*}$, which revealed that $\mathrm{A}+/ \mathrm{AB}^{*}$ training does not necessarily result in enhanced conditioning with $\mathrm{A}$. Indeed, despite the fact that $\mathrm{A}$ and $\mathrm{AB}$ were paired with different USs for this group, there was no hint of an abnormally strong CR during the test trials for this group. The lack of enhanced conditioning with A can be attributed to the conditioning trials with B that took place throughout Stages 1 and 2. According to configural theory (Pearce, 1987, 1994), these conditioning trials will prevent a representation of the $\mathrm{AB}$ configura- 
tion from entering into an excitatory association during Stage 2. A word of caution is needed concerning this conclusion. For all the rats, $\mathrm{B}$ was a clicker and $\mathrm{C}$ was a tone, and it is, therefore, impossible to be certain that the outcome for these groups was due to the experimental treatment, rather than to the different stimuli that were used for $\mathrm{B}$ and $\mathrm{C}$.

The results from Group $\mathrm{A}+/ \mathrm{AB}+$ confirm the results from the group of the same name in Experiment 2. Thus both Experiment 2 and Experiment 3 demonstrate the importance of the change of US for producing enhanced conditioning with $\mathrm{A}$, when conditioning takes place first with $\mathrm{A}$ and then with $\mathrm{AB}$.

\section{GENERAL DISCUSSION}

In each of three experiments, responding during a CS that had been paired with a US was augmented by subsequent conditioning in which the CS was presented in compound with another CS and was followed by a new US. This effect depended on a change in the US between the two stages of conditioning and on the presence of the additional stimulus during the second stage of conditioning. These findings are original.

Throughout this article, we have viewed our findings from the perspective of a configural theory of conditioning. Before we comment further on their significance for this theory, we will examine the implications of our findings for elemental theories of conditioning. According to a theory such as that proposed by Rescorla and Wagner (1972), the changes that occur during conditioning with a compound CS will cease when the sum of the associative strengths of the elements of the compound is equal to a value that is determined by the magnitude of the reinforcer. Given training of the sort $(\mathrm{A}+/ \mathrm{AB}+)$, this theory predicts that, providing conditioning with $\mathrm{A}$ has reached asymptote during Stage 1, there will be no opportunity for the associative strength of this stimulus to increase during Stage 2, and thus, enhanced conditioning with $\mathrm{A}$ is predicted not to occur with this training. The results from Experiments 2 and 3, as well as those of Rescorla (1999) and our unpublished experiments, are consistent with this prediction. The remaining results from our experiments are not so easy to explain with this type of theory. Consider, first, the demonstrations of enhanced conditioning that we found with the $\mathrm{A}+/ \mathrm{AB} *$ treatment. For this outcome to occur, it must be assumed that US* supported a stronger CR than did US+, but in Experiments 2 and 3, the use of reinforcers was counterbalanced, so that for half the subjects, US+ was sucrose and for the remainder, it was food pellets. Given this experimental design, it would not be reasonable to argue, for all the rats in a group, that US+ was less effective than US*. Furthermore, in Experiment 1, the effects of the $\mathrm{A}+/ \mathrm{AB}^{*}$ treatment were compared with a condition in which A was paired first with US+ and then with US*. Although all the subjects received sucrose as US + and food pellets as US*, even if the latter were the more effective reinforcer, the Rescorla-Wagner (1972) model predicts that responding during $\mathrm{A}$ is likely to be stronger, not weaker, after the $\mathrm{A}+/ \mathrm{A}^{*}$ than after the $\mathrm{A}+/ \mathrm{AB} *$ treatment.

Thus, neither an elemental (Rescorla \& Wagner, 1972) nor a configural (Pearce, 1987, 1994) theory is able to explain all of our results. The former can explain the failure to find enhanced conditioning after $\mathrm{A}+/ \mathrm{AB}+$ training, but it is unable to explain the occurrence of this effect after $\mathrm{A}+/ \mathrm{AB} *$ training. In contrast, configural theory can explain our demonstrations of enhanced conditioning with $\mathrm{A}+/ \mathrm{AB} *$ training, but it is unable to explain its absence after $\mathrm{A}+/ \mathrm{AB}+$ training. One possible explanation for our results, therefore, is that rats adopt an elemental strategy when confronted by certain tasks (e.g., $\mathrm{A}+/ \mathrm{AB}+)$ and a configural strategy when confronted by other tasks (e.g., A+/AB*). The obvious problem with this suggestion is that it is not at all clear when rats will adopt one strategy in preference to the other.

In the following discussion, we will pursue an alternative way of explaining our findings, by examining how a particular theory might be modified in order to account for them. We consider, first, an elemental theory and, second, a configural theory. Theories of conditioning typically assume that the asymptotic strength of a CS-US association is determined by the magnitude of the US, without regard to the quality of the US. But if it is assumed that this association somehow contains information about both the magnitude and the specific nature of the US, an elemental theory such as the Rescorla-Wagner (1972) model may be able to explain why we found enhanced conditioning with $\mathrm{A}+/ \mathrm{AB}^{*}$ training. During Stage 1 of this training, an association would develop between $\mathrm{A}$ and a specific representation of US + . The omission of US+ during the $\mathrm{AB}^{*}$ trials of Stage 2 can then be expected to have two consequences. The original A-US+ association will weaken in the same way that any association will weaken through nonreinforcement, and, more important, B might acquire inhibitory properties by virtue of signaling the omission of US+. As these inhibitory properties gain in strength, they will protect the A-US+ association from extinction (Chorozyna, 1962; Soltysik, Wolfe, Nicholas, Wilson, \& Garcia-Sanchez, 1983), so that it may be relatively intact by the end of Stage 2 . Stage 2 will also provide the opportunity for both A and $\mathrm{B}$ to enter into associations with US*. Thus, when A is presented for testing, the removal of $B$ should permit what remains of the A-US+ association to elicit a CR, and the recently acquired A-US* association will also encourage the performance of a CR. Provided that both reinforcers support the same CR, it is possible that the combined influence of these associations will promote a CR of greater than normal strength.

Although the foregoing proposal has the virtue of predicting that $\mathrm{A}+/ \mathrm{AB}^{*}$ training, but not $\mathrm{A}+/ \mathrm{AB}+$ training, will result in enhanced conditioning with $A$, it can be called into question for at least two reasons. The first rests with the proposal that B will serve as an inhibitory CS for US+. If this is correct, pairing B by itself with US+ 


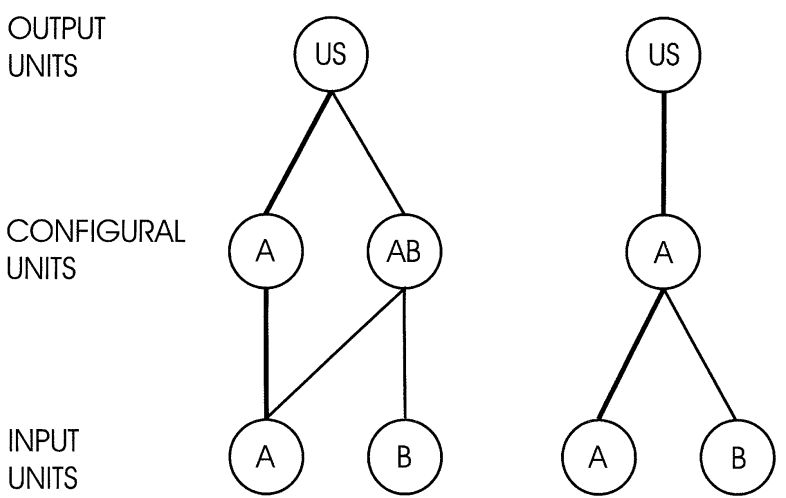

Figure 4. The connections that are predicted to be formed in the connectionist network proposed by Pearce (1994) after animals have received conditioning in which $A$ and then $A B$ are paired with a US (left-hand panel), and a modification to this network (right-hand panel). The width of a connection is intended to depict its relative strength.

should result in relatively slow conditioning (a retardation test for conditioned inhibition; Rescorla, 1969), and presenting B in compound with a CS that has signaled $\mathrm{US}+$ should result in weaker responding than if it is presented in compound with a CS that has signaled US* (a summation test for conditioned inhibition; Rescorla, 1969). We have made a number of attempts to test these predictions in unpublished experiments, and thus far, our efforts have been unsuccessful.

The second reason for calling into question the foregoing explanation comes from studies in which the effect of pairing a single CS with two different appetitive reinforcers in succession has been examined. For example, Rescorla (1996) paired a CS with US+ for a number of sessions and then with US* for a similar number of sessions. The rats then received taste aversion conditioning with US+, which was subsequently found to weaken considerably the strength of responding in the presence of the CS. One explanation for this result is that, when the CS was presented for testing, it was able to activate a representation of US + (and, hence, activate the effects of the taste aversion conditioning), the result being a weak appetitive CR. In other words, the outcome of this experiment strongly suggests that pairing a CS with US+ and then with US* did not weaken the original CS-US+ association (for other experiments that make the same point, see Delamater, 1996; Rescorla, 1997). If this conclusion is accepted, it would be unreasonable to assume for our experiments that there was any tendency for the treatment with $\mathrm{AB}^{*}$ to weaken a previously acquired $\mathrm{A}-$ US + association. In the absence of such a tendency, there would be no opportunity for B to enter into an inhibitory association to protect $\mathrm{A}$ from extinction, and the removal of B for the test stage should not result in evidence of enhanced conditioning with A. Therefore, the explanation that we have offered for our results may, at face value, appear plausible, but it can be called into question on the basis of the results from recent published and unpublished experiments.

Additional findings from Experiment 3 pose a rather different problem for an elemental explanation for our results. During Stage 2 of that experiment, Group $\mathrm{A}+/$ $\mathrm{AB}+$ received intermixed trials with $\mathrm{AB}+$ and $\mathrm{C}+$, and Group $\mathrm{A}+\mathrm{B} * / \mathrm{AB}+$ received intermixed trials with $\mathrm{AB}+$ and $\mathrm{B}+$. According to the Rescorla and Wagner (1972) theory, for Group $\mathrm{A}+\mathrm{B} * / \mathrm{AB}+$, the trials with $\mathrm{B}+$ will block conditioning with A and will result in this stimulus' eliciting a weaker response at the time of testing than for Group $\mathrm{A}+/ \mathrm{AB}+$. There was no hint of support for this prediction, which prompts two possible conclusions. First, the analysis offered by the Rescorla-Wagner theory for the effect of $\mathrm{AB}+\mathrm{B}+$ training after $\mathrm{A}+$ trials, is incorrect. Alternatively, it is conceivable that there were insufficient $\mathrm{B}+$ trials in Stage 2 for Group $\mathrm{A}+\mathrm{B} *$ / $\mathrm{AB}+$. There were, however, $48 \mathrm{~B}+$ trials in Stage 2, which might be considered sufficient to enable this stimulus to have had some impact on the properties of A. Moreover, it is worth noting that these results are not the first occasion on which following $\mathrm{A}+$ trials by $\mathrm{AB}+\mathrm{B}+$ training has been found to have no effect on the properties of $A$. In a series of autoshaping experiments with pigeons, Rescorla (1989) was unable to find any influence at all of the $\mathrm{B}+$ trials on the excitatory properties of A (see, also, Pearce \& Redhead, 1995, Experiment 2). Thus, at the very least, additional experiments are required in order to verify the various assumptions that have been made to allow our findings to be explained from an elemental perspective.

We turn now to a consideration of how configural theory might be modified to explain our results. The network shown in the left half of Figure 4 depicts the connections that are predicted by the theory of Pearce (1994) to result from $\mathrm{A}+/ \mathrm{AB}+$ training. The network has an input layer where units are activated by external stimuli, an intermediate layer comprising configural units, and an output layer. The output layer consists of a single US unit, the activation of which determines the strength of response to a given input pattern. According to Pearce (1994), A+ trials will result in strong connections between the input unit for A and the configural unit for A, and between the configural unit for $\mathrm{A}$ and the output unit. As a result, a strong CR will be performed whenever $\mathrm{A}$ is presented by itself. If $\mathrm{AB}+$ trials are now introduced, the presence of $B$ will restrict the activation of the input unit for A, which, in turn, will restrict the activation of both the configural unit for $A$ and the output unit and will result in a generalization decrement. A further consequence of the introduction of $B$ is that the new pattern of stimulation will encourage the recruitment of a new configural unit, $\mathrm{AB}$, which will be connected to the input units for $A$ and $B$ and to the output unit. The connection between the configural unit for $\mathrm{AB}$ and the output unit will grow until the activations of both config- 
ural units, multiplied by the strength of their connections with the output unit, is able to activate the output unit to a level that is appropriate for the US. If A should again be presented by itself, it will activate its own configural unit maximally and the $\mathrm{AB}$ unit to a lesser extent, with the result that the output unit will be activated to a level that will produce an abnormally strong CR. Thus, as it was originally formulated, the network incorrectly predicts that $\mathrm{A}+/ \mathrm{AB}+$ training will result in enhanced conditioning with $\mathrm{A}$.

One criticism that can be leveled at the network shown in the left-hand panel of Figure 4 is that it is extravagant in its use of configural units. Suppose that the same reinforcer is consistently presented to an animal, but that the stimulation that signals the reinforcer changes slightly from trial to trial. According to Pearce (1994), each change of stimulation will result in a new pattern of activation at the input layer, and hence, a new configural unit will be recruited into the network. A more efficient use of configural units in these circumstances would be to use a single unit to signal the US but to allow this unit to be activated by a variety of similar patterns of stimulation. The network shown in the right half of Figure 4 depicts one way in which this modification may be effected. The following discussion reveals that one consequence of this modification is that it will allow configural theory to predict that training of the $\mathrm{A}+/ \mathrm{AB}+$ sort will not result in enhanced conditioning with A.

When conditioning first takes place with A, strong connections will develop between the input unit for A and the configural unit for $\mathrm{A}$ and between the configural unit for $\mathrm{A}$ and the output unit. If compound $\mathrm{AB}$ should now be introduced and paired with the same US, then, as for the original network, the presence of $\mathrm{B}$ will reduce the degree to which $\mathrm{A}$ is able to activate its input unit and will result in a generalization decrement. Because the US is the same as those for previous trials, instead of the new pattern of stimulation being responsible for the recruitment of a new configural unit, we shall assume that the input unit for B will become connected to the configural unit for A. According to principles proposed by Pearce (1994), the strength of this connection will be relatively weak in order to ensure that the combined presence of $A$ and $\mathrm{B}$ activates the configural unit for A fully and elicits a normal CR. If A should now be presented by itself, it will exert its original influence on the configural unit for $A$ and will again result in a CR of normal strength, rather than in one of supernormal strength. Moreover, given that the strength of the connection between the input unit for B and the configural unit for A is relatively weak, presenting $B$ by itself will result in a rather modest activation of the configural unit and, hence, will result in a weak CR. Thus, the modified network is able to predict blocking, but in keeping with the original network, it follows that blocking will not be complete.

Of course, the proposals that have just been made will be suitable only for conditioning in which different pat- terns of stimulation are followed by the same US. If different patterns are followed by different outcomes, such as the presence or the absence of a US, the network shown in the right-hand panel of Figure 4 leads to the prediction that it would be impossible to discriminate between these patterns. In these circumstances, therefore, the network shown in the left-hand panel of Figure 4 must be used. It is also tempting to speculate that the network in the left-hand panel of Figure 4 determines the outcome of conditioning when different patterns of stimulation (say, A and AB) are followed by different reinforcers (say, food and sucrose). Enhanced conditioning with $\mathrm{A}$ would then be predicted to result from $\mathrm{A}+/ \mathrm{AB}^{*}$ training. If this suggestion is to merit serious consideration, it is necessary to specify precisely how the activation of an output unit is determined.

We have assumed thus far that food and sucrose activate the same output unit. The activity of this unit could, thus, reflect the affective or motivational properties of these reinforcers (Konorski, 1967; Wagner \& Brandon, 1989), or it might reflect the fact that they supported the same CR of magazine approach. On the other hand, because food and sucrose differ in their sensory properties, it is possible that they activate different output units. In order for the modifications to configural theory proposed in the previous paragraph to explain the present (and related) results, we must assume that conditioning results in associations involving both types of output unit: one that reflects the shared properties of the reinforcers, and one that reflects their distinctive properties.

Evidence in support of the suggestion that reinforcers of similar affective value excite the same output unit can be found in a study by Ganesan and Pearce (1988), the design of which was similar to the present experiments, except that food and water were the two reinforcers. Two groups of rats received either $\mathrm{A}+/ \mathrm{AB}+$ or $\mathrm{A}+/ \mathrm{AB} *$ training, followed by test trials with $\mathrm{B}$. Both groups responded similarly during $\mathrm{B}$ and significantly less than did control groups given similar training with $\mathrm{AB}$ but no prior training with A. Thus, the experiment demonstrated blocking. It also demonstrated that the magnitude of this blocking was unaffected by changing the US at the outset of Stage 2 (see, also, Williams, 1994). According to each of the networks shown in Figure 4, blocking in these circumstances is possible only if food and water excite the same output unit.

Once it is acknowledged that the output units referred to in Figure 4 reflect the properties that are held in common between US+ and US*, it is evident that neither network would be able to detect when there has been a change from one reinforcer to the other. For this to be possible, a configural unit must be connected to two output units. One output unit will represent the general characteristics of the reinforcer and, for the method of conditioning used in the present experiments, will determine the strength of the CR that is aroused by a CS. The other output unit will represent the sensory properties of the reinforcer (see, also, Konorski, 1967; Wagner \& Brandon, 
1989). Activation of this unit may have little direct influence on performance, but its presence will determine the specific changes that take place within the network. Providing that the reinforcer presented on any trial corresponds to the properties represented by this second unit, learning will progress according to the network shown in the right-hand side of Figure 4. But if the reinforcer should change during conditioning, it will no longer correspond to the specific representation that is predicted, and learning will develop according to the principles contained within the network shown in the left-hand side of Figure 4.

Although the foregoing modifications of configural theory have been prompted principally by the present results, it is interesting to note that they have implications for other conditioning phenomena, such as acquired equivalence. In an experiment by Honey and Hall (1989), rats received training in which two auditory stimuli signaled food and a third auditory stimulus signaled nothing $(\mathrm{A}+\mathrm{B}+\mathrm{C}-)$. Stimulus $\mathrm{B}$ was then paired with shock. Subsequent test trials revealed a stronger aversive CR in the presence of A than of C. According to the present proposals, the initial training will result in A and B's exciting the same configural unit, providing they share some degree of similarity. The aversive conditioning with B will then result in the configural unit's being associated with shock, and the activation of the configural unit on the test trials with A will excite an aversive CR. Two predictions follow from the proposals developed above for this explanation for acquired equivalence. First, acquired equivalence should not be found when A and B are very different from each other, and second, it should not be found if $\mathrm{A}$ and $\mathrm{B}$ are paired with different appetitive reinforcers during the first stage of training. By conducting experiments that test these and related predictions, it should be possible to evaluate further the admittedly speculative modifications to configural theory that have been developed in this discussion.

The three reported experiments have shown that training of the $\mathrm{A}+/ \mathrm{AB}^{*}$ sort, but not of the $\mathrm{A}+/ \mathrm{AB}+$ sort, can result in enhanced responding during subsequent test trials with A. These results have proved difficult to explain within a single theoretical perspective. In order to explain our results in terms of a configural theory (Pearce, 1987, 1994), it has been necessary to propose a modification to this theory. However, additional experiments are needed in order to evaluate this modification. To reconcile our findings with an elemental analysis of conditioning, we proposed that $\mathrm{A}+/ \mathrm{AB}^{*}$ training results in B's acquiring inhibitory properties by virtue of signaling the absence of US+. Unfortunately, there is no direct evidence that our method of training endowed B with such inhibitory properties. As these issues are addressed, not only will our understanding of the factors that promote abnormally strong conditioning with a CS be improved, but we shall also gain a deeper appreciation of the way in which different representations of the US can influence learning and performance.

\section{REFERENCES}

Chorozyna, H. (1962). Some properties of conditioned inhibition. Acta Biologiae Experimentalis, 22, 5-13.

Delamater, A. R. (1996). Effects of several extinction treatments upon the integrity of Pavlovian stimulus-outcome associations. Animal Learning \& Behavior, 24, 437-449.

Ganesan, R., \& Pearce, J. M. (1988). Effect of changing the unconditioned stimulus on appetitive blocking. Journal of Experimental Psychology: Animal Behavior Processes, 14, 280-291.

Honey, R. C., \& HaLl, G. (1989). Acquired equivalence and distinctiveness of cues. Journal of Experimental Psychology: Animal Behavior Processes, 15, 338-346.

KONORSKI, J. (1967). Integrative activity of the brain. Chicago: University of Chicago Press.

Pearce, J. M. (1987). A model of stimulus generalization for Pavlovian conditioning. Psychological Review, 94, 61-73.

Pearce, J. M. (1994). Similarity and discrimination: A selective review and a connectionist model. Psychological Review, 101, 587-607.

Pearce, J. M., \& Redhead, E. S. (1995). Supernormal conditioning. Journal of Experimental Psychology: Animal Behavior Processes, 21, 155-165.

ResCorla, R. A. (1969). Pavlovian conditioned inhibition. Psychological Bulletin, 72, 77-94.

RESCORLA, R. A. (1971). Variations in the effectiveness of reinforcement and nonreinforcement following prior inhibitory conditioning. Learning \& Motivation, 2, 113-123.

Rescorla, R. A. (1989). Redundant treatments of neutral and excitatory stimuli in autoshaping. Journal of Experimental Psychology: Animal Behavior Processses, 15, 212- 223.

ResCorla, R. A. (1996). Spontaneous recovery after training with multiple outcomes. Animal Learning \& Behavior, 24, 11-18.

ResCorla, R. A. (1997). Spontaneous recovery after Pavlovian conditioning with multiple outcomes. Animal Learning \& Behavior, 25, 99-107.

Rescorla, R. A. (1999). Associative changes in elements and compounds when the other is reinforced. Journal of Experimental Psychology: Animal Behavior Processes, 25, 247-255.

Rescorla, R. A., \& WAGNER, A. R. (1972). A theory of Pavlovian conditioning: Variations in the effectiveness of reinforcement and nonreinforcement. In A. H. Black \& W. F. Prokasy (Eds.), Classical conditioning II: Current research and theory (pp. 64-99). New York: Appleton-Century-Crofts.

Soltysik, S. S., Wolfe, G. E., Nicholas, T., Wilson, W. J., \& GarciaSANCHEZ, J. L. (1983). Blocking of inhibitory conditioning with a serial conditioned stimulus-conditioned inhibitor compound: Maintenance of acquired behavior without an unconditioned stimulus. Learning \& Motivation, 14, 1-29.

WAGNER, A. R. (1971). Elementary associations. In H. H. Kendler \& J. T. Spence (Eds.), Essays in neobehavorism: A memorial volume to Kenneth W. Spence (pp. 187-213). New York: Appleton-CenturyCrofts.

WAGNER, A. R., \& BRANDON, S. E. (1989). Evolution of a structured connectionist model of Pavlovian conditioning (AESOP). In S. B. Klein \& R. R. Mowrer (Eds.), Contemporary learning theories: Pavlovian conditioning and the status of traditional learning theory (pp. 149-189). Hillsdale, NJ: Erlbaum.

WAGNER, A. R., \& RESCORLA, R. A. (1972). Inhibition in Pavlovian conditioning: Application of a theory. In R. A. Boakes \& M. S. Halliday (Eds.), Inhibition and learning (pp. 301-336). London: Academic Press.

WiLliams, B. A. (1994). Blocking despite changes in reinforcer identity. Animal Learning \& Behavior, 22, 442-457.

(Manuscript received June 18, 1998; revision accepted for publication March 23, 1999.) 